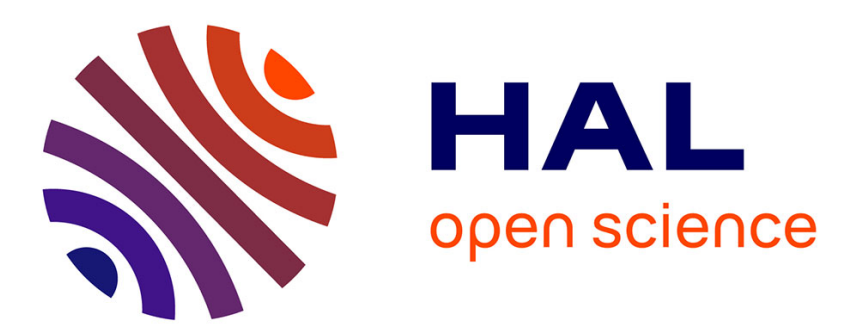

\title{
Négocier à la cour du sultan hafside: le dernier traité de paix conclu avec la Commune de Pise (800/1397)
}

\author{
Mohamed Ouerfelli
}

\section{To cite this version:}

Mohamed Ouerfelli. Négocier à la cour du sultan hafside: le dernier traité de paix conclu avec la Commune de Pise (800/1397). Frédéric Bauden. Culture matérielle et contacts diplomatiques entre l'Occident latin, Byzance et l'Orient islamique (XIe-XVIe siècle), 83, Brill, pp.447-463, 2021, Islamic History and Civilization, 978-90-04-46533-6. 10.1163/9789004465381_016 . hal-03277029

\section{HAL Id: hal-03277029 https://hal.science/hal-03277029}

Submitted on 17 Jan 2022

HAL is a multi-disciplinary open access archive for the deposit and dissemination of scientific research documents, whether they are published or not. The documents may come from teaching and research institutions in France or abroad, or from public or private research centers.
L'archive ouverte pluridisciplinaire HAL, est destinée au dépôt et à la diffusion de documents scientifiques de niveau recherche, publiés ou non, émanant des établissements d'enseignement et de recherche français ou étrangers, des laboratoires publics ou privés. 


\section{Négocier à la cour du sultan hafside : \\ le dernier traité de paix conclu avec la Commune de Pise (1397)}

Mohamed OUERFELLI

Aix Marseille Univ, CNRS, IREMAM, Aix-en-Provence, France

\section{Introduction}

Pour se consacrer pleinement au front ibérique, l’empire almohade a établi depuis la seconde moitié du $\mathrm{XII}^{\mathrm{e}}$ siècle des relations pacifiques avec les républiques maritimes italiennes, fondées sur l'échange, dans le cadre de traités de paix et de commerce conclus entre les deux parties. Les Pisans, qui étaient les premiers à avoir signé un traité de paix avec les Almoravides, en $1133^{1}$, fréquentaient les ports maghrébins et ont négocié les premiers accords avec les Almohades ${ }^{2}$. Leur présence dans la Méditerranée occidentale et en Ifrīqiya plus particulièrement a connu des moments forts au XIII ${ }^{\mathrm{e}}$ siècle. Les Pisans ont régulièrement renouvelé les traités de paix avec les pouvoirs maghrébins successifs, mais cette assiduité s'estompe progressivement avec l'effacement de la puissance pisane après la défaite de la Meloria en 1284 contre sa rivale Gênes et les nombreux conflits qui l'ont opposée à la puissance montante de Florence, notamment à partir de $1381^{3}$. À la fin du XIV ${ }^{\mathrm{e}}$ siècle, en grande difficulté, les Pisans peinent à retrouver leur place d'antan ; ils réussissent tout de même, après plusieurs années de négociations, à conclure un nouveau et dernier traité avec les Hafsides, le 14 décembre 1397. Le contexte de la conclusion de cet accord mérite d’être analysé finement, pour mieux comprendre les enjeux majeurs des relations diplomatiques et commerciales entre Pisans et Hafsides, qui cherchent à défendre leurs intérêts respectifs.

Ce traité, original par rapport aux précédents, est riche en enseignements sur les modalités de négociations et sur le fonctionnement des chancelleries hafside et pisane, où travaille un personnel diplomatique nombreux, dans l'objectif de mener à bien les négociations, accompagner les ambassadeurs, subvenir à leurs besoins, rédiger et traduire les actes officiels et les comptes rendus des audiences. Il n’a pourtant pas attiré tout l'intérêt qu’il

\footnotetext{
${ }^{1}$ Gentile, Gli annales pisani 9.

${ }^{2}$ Ouerfelli, Personnel diplomatique 122.

${ }^{3}$ Doumerc, Venise 32.
} 
mérite depuis sa publication par Michele Amari et sa réédition par Louis de Mas Latrie ${ }^{4}$. Les travaux se sont focalisés sur les informations d'ordre commercial et politique que comporte ce traité $^{5}$, mais à aucun moment sur son support, ses différentes versions et les copies qui en ont été faites, ce à quoi je m'attèle dans cette contribution. Il s'agira dans un premier temps de cerner le contexte dans lequel a été signé le traité de 1397 ; seront examinées ensuite les modalités de négociations entre Pisans et Hafsides et la conclusion finale du traité. Il sera question en dernier lieu de la présentation matérielle du traité, de ses différentes versions, arabes, latine et italiennes et de leur articulation ${ }^{6}$.

\section{1- Le contexte : les difficultés de la seconde moitié du XIV siècle}

Si Pise a éprouvé de grandes difficultés depuis les années 1370 à renouveler son traité de paix avec le pouvoir hafside, l'expédition de Djerba à laquelle ont participé des armateurs pisans, en 1388, a constitué un obstacle supplémentaire pour la signature d'un accord garantissant aux ressortissants de la Commune de Pise la fréquentation des ports hafsides sans rencontrer de problèmes majeurs. Mais la crise semble beaucoup plus profonde dans les relations entre les deux États ; elle remonte aux années 1370 lorsque Pise a essayé de négocier avec tous les prétendants au pouvoir hafside à Bône, à Bougie, à Tunis, qui se livraient une lutte acharnée pour dominer l'Ifrīqiya. Une série de quatre documents rédigés sur parchemin et produits par la chancellerie pisane, à la date du 11 septembre 1378, porte sur une mission ô combien délicate pour confirmer et conforter les privilèges accordés aux Pisans depuis plusieurs siècles. Pour ce faire, Piero Bullia de Gualandi est mandaté par le gouvernement pisan auprès des pouvoirs autonomes des trois villes hafsides, afin de négocier avec eux, sans avantager une quelconque partie ${ }^{7}$.

La multiplication des actes de piraterie dans la Méditerranée occidentale n’a pas non plus arrangé les affaires pisano-maghrébines ${ }^{8}$. Sous le commandement de l'amiral Muhammad Ibn al-Mahdī (mort en 1402) ${ }^{9}$, pirates et corsaires, bougiotes notamment, ont redoublé d'activité, sans doute en réponse à une conjoncture économique déprimée, où le port de Bougie peine à

\footnotetext{
${ }^{4}$ Amari, I diplomi 123-136 (texte arabe) et 319-325 (texte italien) ; Mas Latrie, traités 70-87.

${ }^{5}$ Banti, I trattati 65-69 ; Doumerc, Venise 32.

${ }^{6}$ Ce document fera l'objet d'une édition critique et d'une traduction dans une publication en préparation.

${ }^{7}$ Archivio di Stato di Pisa (désormais ASP.), Diplomatico, Atti Pubblici, 16 septembre 1378 ; Amari Diplomi, 313 ; Bensaci, Pise et le Maghrib 179.

${ }^{8}$ Dolores Lopez, La Corona 158-159 ; Valérian, Les relations 86-87.

${ }^{9}$ Ibn al-Qunfud, Al-Fārisiya 199 ; Valérian, Bougie 453-454.
} 
reprendre son dynamisme du $\mathrm{XIII}^{\mathrm{e}}$ siècle, beaucoup plus que par volonté de retirer des avantages politiques ou diplomatiques à l'encontre des puissances chrétiennes ${ }^{10}$. Plusieurs documents conservés dans l'Archivio di Stato di Pisa montrent que de nombreux Pisans ont été capturés par des corsaires et ont passé de longs mois, voire des années dans les geôles de Bougie. Des notaires majorquins ont rédigé, en 1379, des reconnaissances de dettes de captifs libérés envers maître Andrea di Barruccio, pisan, présent à Majorque, vraisemblablement dans l'objectif de payer la rançon des captifs pisans ${ }^{11}$. Un document daté du 6 septembre 1382, signalé dans l'inventaire du Diplomatico, dans la série des Atti publici, mais qui manque dans les archives de Pise, détaille les modalités de libération des captifs de Pise et de Piombino, retenus en Berbérie, par le même Andrea di Barruccio ${ }^{12}$. Ibn Khaldūn se fait l'écho du développement considérable de cette activité, plus particulièrement à Bougie, enrichie par le butin et les esclaves capturés sur les côtes provençales et italiennes, avec la complicité du pouvoir central de Tunis, resté silencieux malgré les plaintes répétées des puissances chrétiennes ${ }^{13}$. La recrudescence de la course et de la piraterie a incité ces dernières à exercer des représailles contre les Hafsides, actions militaires auxquelles les Pisans se sont joints volontiers. Ces derniers, qui n’obtiennent aucun résultat de la tournée de Piero Bullia de Gualandi, se rallient, en 1379, aux Aragonais pour piller le littoral maghrébin ${ }^{14}$.

Le statu quo observé dans les relations avec les Hafsides décide les Pisans à accepter de participer de nouveau, aux côtés de leurs ennemis génois cette fois-ci, à une expédition de grande envergure contre l'Ifrīqiya, en 1388, pour faire plier Abū al-Abbās (1370-1394), en train d'affirmer son pouvoir en réunifiant le domaine hafside, et l'obliger à cesser de soutenir la course et à négocier un nouveau traité de paix.

Des lettres envoyées par le doge de Gênes à son homologue vénitien, entre janvier et avril 1388, indiquent clairement la participation de la République de Pise à une coalition dirigée contre le sultanat hafside, et sa disposition à envoyer deux galées armées ${ }^{15}$. Cette participation est revue à la hausse, puisque ce sont cinq galées commandées par Francesco Orlandi qui prennent part à l'expédition de Djerba ${ }^{16}$. Si le rôle de Pise est nettement moins important que celui de Gênes qui envoie pas moins de quinze galées et six autres navires (ligneta), il n’en

\footnotetext{
${ }^{10}$ Valérian, Bougie 451-458.

${ }^{11}$ ASP. Diplomatico, Atti publici 16, 17 et 19 septembre 1379 (huit documents au total).

${ }^{12}$ Inventaire manuscrit 150.

${ }^{13}$ Ibn Khaldūn, Tārīkh VI, 578-579.

${ }^{14}$ Brunschvig, Berbérie I, 196-197.

${ }^{15}$ Mas Latrie, Traités 129.

${ }^{16}$ Roncioni, Delle istorie 946 ; Mas Latrie, Traités 239.
} 
demeure pas moins que les Pisans apparaissent désormais aux yeux des Hafsides comme des ennemis, qui ont agi à l'encontre des intérêts des deux pays, d'où leur réticence, voire leurs tergiversations à signer la paix avec les Pisans, largement dépassés par le dynamisme affiché par leurs rivaux les Florentins, qui ont réussi à séduire les Hafsides, notamment par la solidité de leur monnaie et sa diffusion dans toute la Méditerranée ${ }^{17}$. Les Pisans semblent donc démunis face aux Hafsides, contrairement aux Génois, en position de force dès lors qu’ils ont pris la tête des deux expéditions. Les Vénitiens en revanche, ont finalement renoncé à participer à cette coalition, non sans arrières-pensées. Par cette position, ils affichent leur neutralité dans ce conflit et profitent des situations délicates des Génois et des Pisans, pour s’imposer comme étant les interlocuteurs privilégiés du pouvoir hafside ${ }^{18}$.

L'expédition, soutenue par le pape Urbain VI et à laquelle a participé activement l'amiral sicilien Manfredi Chiaramonte ${ }^{19}$, n’a pas donné les résultats escomptés, malgré le pillage et l'occupation de Djerba pendant plusieurs mois ${ }^{20}$. C'est ce qui explique l'organisation d'une deuxième campagne militaire franco-génoise, en 1390, dirigée cette fois-ci contre la ville de Mahdiya, qui abrite des Génois commerçant avec l'Ifrīqiya ${ }^{21}$. Il n'est pas question en revanche de s'aventurer devant la capitale hafside ; le souvenir de la croisade de Louis IX est encore vivace. Comme la précédente, cette expédition, s’est soldée par un échec ${ }^{22}$, dans la mesure où elle n’a pas réussi à réduire la force de frappe des corsaires maghrébins, qui pillent Syracuse en 1393 et vont jusqu’à capturer son évêque ${ }^{23}$.

\section{2- Les négociations et la conclusion du traité de paix}

Une fois la tension baissée d'un cran entre les Hafsides et les villes maritimes italiennes, Gênes et Venise sont revenues rapidement négocier avec Abū al-'Abbās, mais la Commune de Pise est restée quelque peu à l'écart ; distancée par ses rivaux, la cité toscane tente vaille que vaille de se rapprocher du souverain hafside. Pour cela, Giacomo d’Appiano, fraîchement élu à la tête du gouvernement pisan, dépêche en 1393 Niccolò Lanfreducci, juriste et homme

\footnotetext{
${ }^{17}$ Doumerc, Venise 32-33 ; Michienzi, Datini 166-169.

${ }^{18}$ Brunschvig, La Berbérie I, 197-198 ; Doumerc, Venise 40-41.

${ }^{19}$ Anonyme, Cronica volgare 69 ; Brunschvig, La Berbérie I, 198 ; Bresc, Un monde 829.

${ }^{20}$ Anonyme, Cronica volgare 69 ; Brunschvig, La Berbérie I, 198.

${ }^{21}$ Ibn Khaldūn, Kitāb al-'ibar VI, 578-579 ; Ibn al-Šammā', Al-'adilla 111.

${ }^{22}$ Ibn Khaldūn, Kitāb al-‘ibar VI, 578-579.

${ }^{23}$ Histoire générale de la Tunisie II, 380.
} 
de lettres ${ }^{24}$, en qualité d'ambassadeur, pour se rendre à Tunis, afin d'expliquer au sultan hafside ce qui s’est réellement passé selon le point de vue des autorités pisanes et demander le renouvellement des privilèges accordés aux sujets de la République ${ }^{25}$.

Hormis la lettre d'accréditation délivrée par les autorités pisanes à Niccolò Lanfreducci, lui fixant le cadre de sa mission, nous ne connaissons rien de son séjour à Tunis, ni sa durée : a-t-il été accueilli par le sultan hafside ou seulement par son représentant ? Comment se sont déroulées les négociations ? Quelle réponse a-t-il obtenu à l’issue de ses entretiens avec le pouvoir hafside ? Autant de questions qui restent sans réponse du fait du silence à la fois des sources hafsides et pisanes. Le rapport rédigé par l'ambassadeur vénitien Giacomo Valaresso et envoyé à Venise lors de négociations en vue de renouveler la paix entre la Sérénissime et le sultan hafside Abū al-'Abbās, en 1391, nous éclaire sur la position de celui-ci envers les Vénitiens, mais aussi envers ceux qui ont participé aux attaques contre les possessions hafsides, entre autres les Pisans ${ }^{26}$.

Quoi qu'il en soit, la mission diplomatique de Niccolò Lanfreducci, difficile en réalité, ne semble pas avoir été couronnée de succès ; le traité de paix tant espéré n’a pas été conclu. Les arguments apportés par les Pisans, comme l'indique la lettre de mission de l'ambassadeur, quant à leur implication dans l'expédition de Djerba, n’ont sans doute pas convaincu les Hafsides :

In caso che lo dicto signore re dicesse, li Pisani essere andati con loro galee et legni armati contro di lui et sua gente, lo dicto ambasciadore saviamente, presa la licentia del rispondere, risponda che la verità è che certi citadini di Pisa, li quali erano gravemente offesi da' suoi armatori et socto posti, per ricoverare et ristorare li danni a loro facti, armorno con loro soldati, et con soldati del magnifico signore et amiraglio Manfredi Chiaramonte, due galee, sensa alcuno soldo o contributione de' prefati signori et comune di Pisa $^{27}$.

L'échec des négociations avec Abū al-'Abbās est synonyme d'une rupture des relations entre celui-ci et les Pisans, qui étaient jusqu'au milieu du $\mathrm{XIV}^{\mathrm{e}}$ siècle nombreux et très appréciés dans les ports maghrébins, et menaient d'importantes affaires. Les guerres contre Florence, la rivalité avec Gênes et l'implication de la Couronne d'Aragon dans les

\footnotetext{
${ }^{24}$ Tronci, Memorie 477.

${ }^{25}$ Mas Latrie, Introduction 243 ; Ouerfelli, Les ambassadeurs 104.

${ }^{26}$ Mas Latrie, Introduction 246 ; Doumerc, Venise 41-42.

${ }^{27}$ Amari, I Diplomi 318.
} 
affaires maghrébines ont sensiblement affaibli la Commune, qui n’a plus les moyens, y compris militaires, de défendre ses intérêts et d’infléchir la position du sultan hafside, décidé à se passer des services des Pisans ${ }^{28}$. Signe évident de l'affaiblissement de leur présence en Ifrīqiya, les fondouks dont ils disposaient se sont dégradés et sont tombés en ruine; les autorités hafsides ne les surveillent plus ${ }^{29}$. Il faut attendre la mort d'Abū al-'Abbās en 1394 et l'avènement de son fils Abū Fāris 'Abd al-'Azīz (1394-1434) pour observer un net réchauffement et une reprise progressive des relations diplomatiques.

Trois ans plus tard, soit le 14 décembre 1397, les deux parties parviennent à mettre fin aux hostilités et à conclure un traité de paix et de commerce. Ce document, notre seule et unique source conservée en plusieurs versions, nous renseigne sur l'identité de l'ambassadeur pisan, les modalités de négociations et le contenu précis de l'accord. La Commune de Pise a visiblement changé de stratégie cette fois-ci : plus question d'envoyer un juriste ou un homme de lettres pour convaincre les Hafsides, mais un homme d'affaires en relation étroite avec les places portuaires maghrébines. Andrea di Michele del Campo est nommé à la tête de l'ambassade pisane pour négocier avec le sultan le nouveau et ultime accord de paix et la libération des prisonniers pisans. Qualifié de tăğir dans la version arabe du traité et de mercante dans la traduction italienne, Andrea di Michele del Campo est un homme d'affaires qui connait parfaitement les réalités maghrébines; il dispose de réseaux commerciaux solidement implantés dans le Maghreb, en relation notamment avec la Sicile où s'est installée une partie de la famille des $\mathrm{Campo}^{30}$; des atouts qui lui permettent de convaincre et de négocier la signature de la paix ${ }^{31}$.

D’après notre traité, l'ambassadeur pisan s'est présenté devant les autorités hafsides muni d'une lettre de mission rédigée par la main du notaire public Jacopo di Nocco da Cascina le premier juin 1397 :

Ut de ejus procura constat publico intrumento, manu Jacobi quondam

Nocchi de Cascina, civis Pisani, publici notarii, scriptum Dominice incarnationis anno 1397, indictione quinta, die primo Junii, secundum cursum et consuetudinem Pisanorum ${ }^{32}$.

Ce mandat comporte des instructions précises lui fixant les limites de son pouvoir et les prérogatives de négocier et de ratifier un traité au nom du capitaine Giacomo d’Appiano et

\footnotetext{
${ }^{28}$ Banti, I trattati 65.

${ }^{29}$ Clause $n^{\circ} 4$ du traité du 14 décembre 1397.

${ }^{30}$ Petralia, Banchieri 156-159.

${ }^{31}$ Ouerfelli, Les ambassadeurs 105.

${ }^{32}$ ASP. Diplomatico, Atti publici, Comune A 27, f. 1 r ${ }^{\circ}$; Mas Latrie, Traités 71.
} 
de la Commune de Pise. Le document en question est écrit en latin et porte la marque habituelle ou la signature des Pisans selon ce qui est indiqué dans le traité. Il a fait l'objet d'un examen et d'une authentification par la chancellerie hafside ; pour cela, elle a fait venir des traducteurs, mais aussi les consuls et les marchands occidentaux présents à Tunis, les invitant à témoigner de l'identité de l'ambassadeur et de l'authenticité de son mandat et de la procuration qu'il a présentés. Cette procédure préalable à toute négociation n’est pas due comme on pourrait le penser à la méfiance des Hafsides vis-à-vis des Pisans et aux rapports tendus entre les deux États, mais elle semble être la règle appliquée par la chancellerie hafside. Le traité conclu en 1353 comporte ces mêmes dispositions d'identification des lettres présentées par l’ambassadeur pisan Ranieri Porcellino ${ }^{33}$ :

Il a présenté une procuration écrite en latin ('ăgmă), qu'il a été invité à lire dans l'heureux dīwān de la capitale susdite avec l'autorisation du responsable, le šeykh [...] al-'Abbās Ahmad Ibn Tafrāghīn [...] Des marchands chrétiens, leurs consuls et leur prêtre, aidés par les traducteurs musulmans, ont déclaré qu'il s'agissait bien d'une procuration authentique et qu'il n'y avait pas de doute qu'ils utilisaient ce modèle entre eux ${ }^{34}$.

Si l’on excepte le traité de 1353 conclu avec Pise et négocié par le puissant chambellan ( $h \bar{a} \bar{g} i b$ ) Ibn Tafrāğ̄inn, qui détenait la réalité du pouvoir en Ifrīqiya ${ }^{35}$, ce sont toujours les sultans hafsides qui disposent des prérogatives de signer les accords de paix avec les puissances étrangères ; c’est un acte de souveraineté par excellence. Fait nouveau, ce n’est pas le sultan Abū Fāris, qui règne pourtant en maître, qui a négocié et ratifié le traité de 1397, mais le šeykh almohade Muhammad Ibn Abī Hilāl. Ce fidèle compagnon d'Abū al-'Abbās, d'Abū Fāris et de 'Uthmān (1435-1488), a accompli une longue et riche carrière ; il s'est vu confier la tâche de mener la politique étrangère du sultanat. Il a négocié et ratifié, au nom du souverain, des traités de paix avec les Génois ${ }^{36}$, les Vénitiens ${ }^{37}$, les Catalans ${ }^{38}$ et les Pisans, qui ont perdu du terrain sur les échelles maghrébines au profil de leurs concurrents, notamment les Florentins.

\footnotetext{
${ }^{33}$ Ouerfelli, Les ambassadeurs 101.

${ }^{34}$ Amari, I Diplomi 125 ; Mas Latrie, Traités 72 ; Ouerfelli, Les ambassadeurs 102.

${ }^{35}$ Amari, I Diplomi 98-111 (version arabe) et 303-308 (version latine) ; Mas Latrie, Traités 55-65 ; Brunschvig, La Berbérie I, 181.

${ }^{36}$ Mas Latrie, Traités 130 ; Brunschvig, La Berbérie I, 197 : traité de 1383 confirmé en 1391 par Gentile de Grimaldi et Luchino de Bonavey, ambassadeurs génois.

${ }^{37}$ Mas Latrie, Traités 232-237 ; Girardi, Venezia 43-49 ; Brunschvig, La Berbérie I, 203-204 (traité de 1392).

${ }^{38}$ Brunschvig, La Berbérie I, 224 (traité de 1403).
} 
Ce traité, s’il ne diffère pas dans la globalité de ses clauses des accords précédents, est tout de même original à plus d'un titre ; il comporte deux clauses totalement inédites. La première se rapporte à la durée de validité du traité. Celui-ci est durable et perpétuel (sulhun mustamirrun 'alā al-dawāmi), ce qui contraste littéralement avec les principes du droit musulman. Les accords de paix signés avec les puissances chrétiennes sont des trêves et doivent être limités dans le temps ${ }^{39}$. Le traité de 1234 est signé pour trente ans ${ }^{40}$; celui de 1264 est valable vingt ans ${ }^{41}$. La durée de validité des traités conclus en 1313, en 1353 et en $1358^{42}$, est limitée en revanche à seulement dix ans. Cette durée est la norme appliquée généralement par les souverains musulmans, en référence au traité de Hudaybiya conclu en 628 entre le Prophète et les Quraychites de la Mecque ${ }^{43}$. Comme on le constate avec les premiers traités hafsides et surtout le dernier conclu avec les Pisans, les considérations religieuses ont peu à peu cédé la place au réalisme économique, dont fait preuve le sultan hafside Abū Fāris, désireux de consolider son pouvoir à l'intérieur et ses relations avec les puissances chrétiennes.

La seconde clause inédite engage la responsabilité des consuls pisans en cas d'attaque opérée par des citoyens de la Commune contre les intérêts du sultanat :

Item, quod nullus Pisanus in portubus terrarum dicti regis audeat vel presumat alicui dannum inferre, quia tunc pro omnibus consul et sive consules Pisanorum puniretur sive punirentur ${ }^{44}$.

Si la rédaction arabe ne désigne pas expressément les consuls, contrairement aux textes latin et italien, les personnes susceptibles de collaborer et de défendre les intérêts pisans auprès des autorités hafsides, notamment la douane, sont les consuls en premier chef.

Si une galée ou un lignum appartenant à des Pisans ou à leurs alliés est sorti en mer pour croiser ou causer des dommages aux musulmans, il incombe aux Pisans de les capturer et de tuer leurs occupants. Leur argent doit être extorqué où qu'il soit, à Pise ou à sa juridiction et remis à la douane. Et s'ils ne peuvent pas le faire eux-mêmes, ils doivent remettre leurs avoirs à la dite douane.

\footnotetext{
${ }^{39}$ Ibn 'Abd al-Barr, Al-Kāfì fì fiqh 210 ; al-'Umarī, Al-ta 'rīf 211-214 ; al-Rassā', Šarh I, 226 ; Bauden, Due trattati 59.

${ }^{40}$ Amari, I Diplomi 292 ; Ouerfelli, Les enjeux commerciaux 210.

${ }^{41}$ Amari, I Diplomi 295 ; Ouerfelli, Les enjeux commerciaux 210.

${ }^{42}$ Ce traité est négocié entre le Mérinide Abū 'Inān (1348-1358) et la Commune de Pise ; Amari, I Diplomi 312 ; Ouerfelli, Les enjeux commerciaux 210.

${ }^{43}$ Al-Qāḍ̂̄ al-Nu'mān, Da 'ā'im I, 379 ; Id., Kitāb al-’iqtiṣār 54 ; Hamidullah, Documents 34-35 et doc. n 4, 1415 ; Kadduri, War and Peace 210-2013 et 249-250.

${ }^{44}$ ASP. Diplomatico, Atti publici, Comune A 27, f. 5 v ; Mas Latrie, Traités 84.
} 
Avec ces deux clauses, le traité de 1397 bouleverse les traditions diplomatiques en vigueur et les normes juridiques en fonction desquelles étaient jusque-là négociés les accords de paix. Il tranche avec les précédents, ignore le caractère temporaire des trêves selon le droit musulman et révèle la position délicate de la Commune de Pise dans ses démarches pour renouveler la paix avec le sultan hafside.

\section{3- Les différentes versions du traité}

Le traité du 14 décembre 1397 nous est parvenu dans sa version originale arabe ; par ailleurs, il en existe également une copie, rédigée postérieurement, mais qui n’est pas attestée par des témoins comme il est écrit au début du document :

Ceci est la copie d'un traité de paix signé dans la capitale ; il a été rédigé dans la mosquée. Elle n'est pas attestée par des témoins ${ }^{45}$.

La rédaction originale, comme la copie, sont exécutées sur parchemin, avec une écriture claire dite magribi $\bar{l}$, dont les principales caractéristiques résident dans la notation des lettres $f \bar{a}^{\prime}$ avec un point diacritique sous la lettre, et le $q \bar{a} f$ avec un point diacritique sur la lettre $^{46}$.

$$
\text { Lettre } f \bar{a}^{\prime} \text { (و أجفان) }
$$

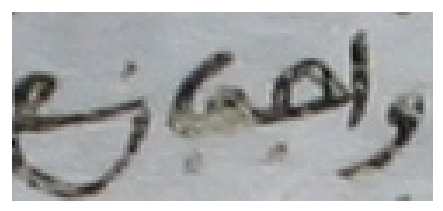

Lettre $q \bar{a} f(و د)$

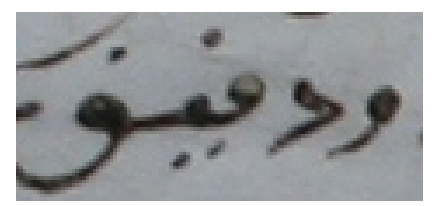

L’original porte des traces d'usure, bien perceptibles sur certains passages. Il se démarque de la copie par des précisions supplémentaires, notamment en ce qui concerne la mention de la langue dans laquelle est rédigé le traité, soit l’arabe. Sont également ajoutées quelques clauses, qui ne figurent pas dans la copie, ainsi que l'indication des noms de deux témoins et leurs signatures.

\footnotetext{
${ }^{45}$ Archivio di Stato di Pisa, Diplomatico, Atti Pubblici, 14 décembre 1397.

${ }^{46}$ Bauden, Due trattati 41.
} 


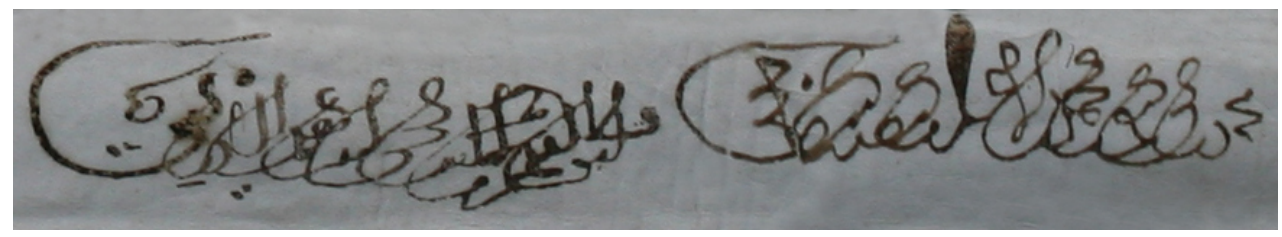

Le traité comporte vingt-six clauses, mais contrairement à ce que l'on peut attendre, après la citation des témoins et l'indication de la date et du lieu de conclusion du traité, d'autres clauses ont été ajoutées, sans doute à la demande de l'ambassadeur pisan, preuve que les négociations se sont poursuivies au moment de la rédaction du traité. Aux deux clauses supplémentaires, s’ajoutent d'autres indications sur les taxes perçues par la douane, mais qui sont courtes et manquent de précisions. Les ajouts et parfois les ratures montrent que le scribe rédigeait le texte au moment où les discussions étaient en cours et il semble qu'il n’a pas eu le temps d'écrire un brouillon avant de mettre son texte au propre ; l'empressement des Pisans à conclure ce traité y est peut-être pour quelque chose. Qu'importe la forme du moment que ce document permet de garantir aux Pisans d’ouvrir une nouvelle page dans leur relation avec les Hafsides, de plus en plus tournés vers de nouveaux acteurs plus puissants, tels les Florentins.

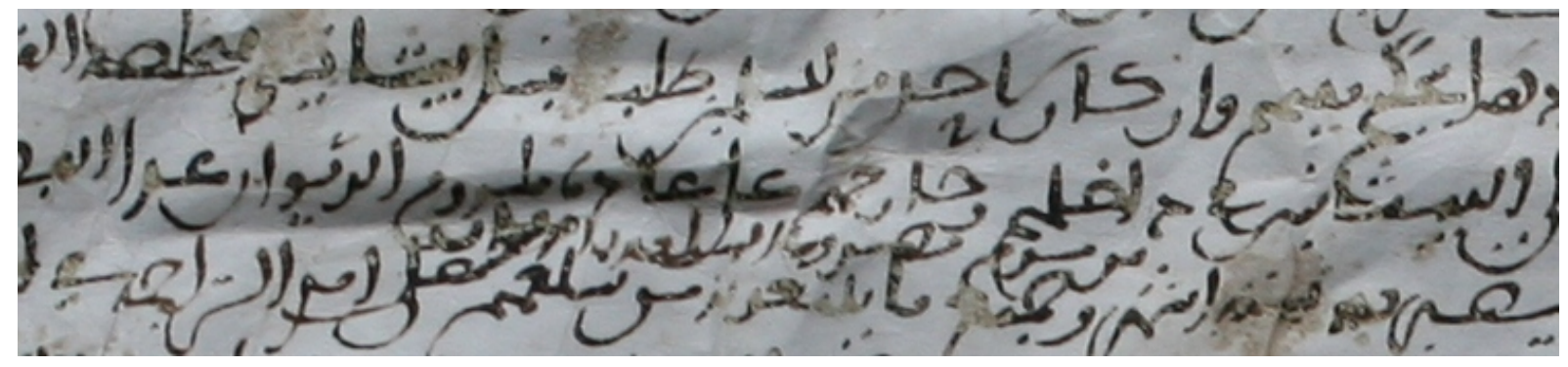

De l’original arabe devait être exécutée immédiatement, soit sur le lieu des négociations, une traduction latine du traité, sur parchemin, comme le document arabe ; la traduction latine l'indique clairement :

Toutes les choses susdites ont été interprétées et traduites par les traducteurs de la langue arabe et sarrasine en latin et du latin dans la langue arabe et sarrasine par Piero Paganucci, citoyen pisan habitant à Tunis, traducteur au fondouk des dits Pisans ${ }^{47}$.

Pourquoi le document latin, fruit d'une traduction du texte original arabe, est-il traduit de nouveau et immédiatement en arabe en présence de l'ambassadeur pisan ? La réponse est sans doute à chercher dans le contexte d'une relation bilatérale tendue, où les Hafsides sont de

\footnotetext{
${ }^{47}$ ASP. Diplomatico cartaceo 1398 Dicembre 14.
} 
plus en plus méfiants à l'égard des Pisans. La rétro-traduction du texte latin, confiée en général à une tierce personne, servait certainement à effectuer une vérification du contenu de l'accord par les autorités hafsides, afin d'éviter tout malentendu dans la lecture des clauses du traité, ce qui porte à croire à une certaine méfiance vis-à-vis des Pisans entre autres. Pour lever toute ambigüité, les sultans hafsides, en particulier Abū Fāris (1394-1434), confient la traduction des lettres qu'ils reçoivent à plusieurs traducteurs, comme il ressort du témoignage d’Anselme Turmeda ('Abd Allâh al-Turğumān) ${ }^{48}$.

À partir de la traduction latine, plusieurs exemplaires devaient être produits, dont un revenait au sultan hafside et les autres à la Commune de Pise. Cette traduction originale est aujourd'hui introuvable et il ne reste que des exemplaires recopiés postérieurement: une version latine et trois italiennes ${ }^{49}$. Elles sont conservées actuellement dans le Diplomatico, Atti Publici, dans un registre factice, dont la reliure date vraisemblablement du XIX ${ }^{\mathrm{e}}$ siècle. Les trois exemplaires italiens suivent celui en latin ; je les appellerai A, B, C, D en suivant l'ordre du registre. Les quatre copies sont rédigées dans une écriture de style mercantesca, de la fin du $\mathrm{XIV}^{\mathrm{e}}$ ou du début du $\mathrm{XV}^{\mathrm{e}}$ siècle. Les documents $\mathrm{A}$ et $\mathrm{B}$ ont été exécutés par la même main comme on peut l'observer dans les deux extraits suivants :

Exemplaire A (texte latin)

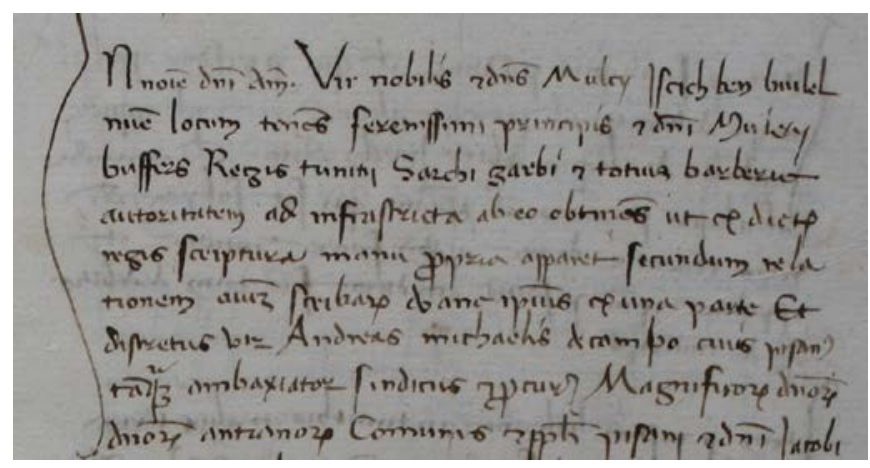

Exemplaire B (texte italien)

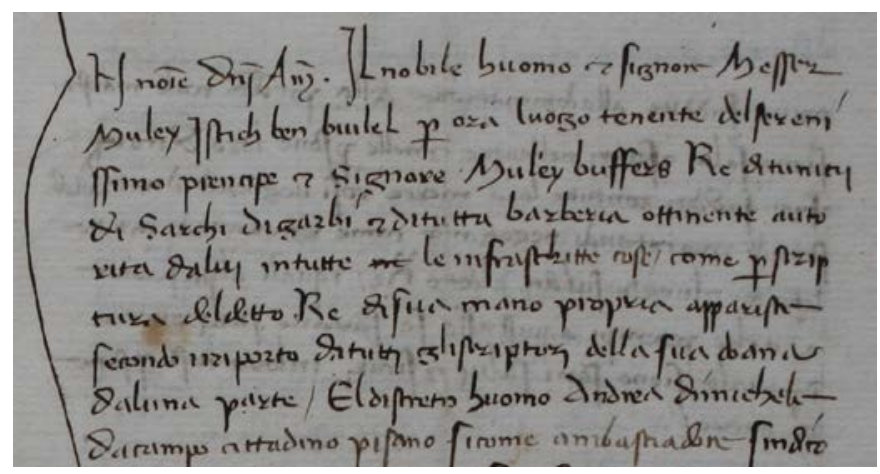

\footnotetext{
48 'Abd Allâh al-Turğumān 79-80.

${ }^{49}$ ASP. Diplomatico cartaceo 1398 Dicembre 14.
} 
Si le format des versions $\mathrm{A}, \mathrm{B}$ et $\mathrm{D}$ est à peu près le même, le document $\mathrm{C}$ a en revanche un format original de type oblong. Cet exemplaire était sans doute destiné à être transporté. Rédigés sur papier, ces quatre exemplaires ne sont visiblement pas des copies officielles, mais plutôt des duplicatas produits pour l'usage de l'administration pisane, voire des hommes d'affaires en relation avec le sultanat hafside, pour les montrer en cas de litige avec les fonctionnaires de la douane ou avec des marchands sujets du sultan.

Des différences entre les versions du traité sont également perceptibles dans le contenu. La version arabe consacre une place prépondérante à la titulature du sultan hafside, qui se déploie dans cet acte pour rappeler sa légitimité par sa filiation et par l'exercice du pouvoir en qualité de calife, d’imām et d’Émir des croyants. Les Pisans en revanche sont animés par des considérations commerciales plutôt que par des clauses d’ordre politique.

Le traité met l'accent sur la nécessité de lutter contre la course et la piraterie ; de nombreuses clauses sont consacrées à cette question épineuse qui entrave les échanges commerciaux. Mais il comporte également des différences notables entre les rédactions et leurs traductions. Seules les versions latine et italiennes précisent que les victuailles importées par les Pisans en terre hafside pour leur propre consommation ne payent que la moitié des droits de douane, qui sont de l'ordre de $10 \%{ }^{50}$. Une clause figurant dans le texte arabe original évoque les transactions financières faites par procuration au profit des Pisans à Tunis, mais elle ne précise pas que les documents doivent être rédigés en latin, puis traduits en arabe, comme le soulignent les exemplaires latin et italiens ${ }^{51}$.

\section{Conclusion}

Ce dernier traité de paix et de commerce conclu visiblement à la hâte en 1397, entre le sultan hafside Abū Fāris 'Abd al-'Azīz et le capitaine de Pise Giacomo d'Appiano, par l'intermédiaire de l'ambassadeur Andrea di Michele del Campo, est non seulement riche par son contenu, notamment en ce qui concerne la précision des clauses commerciales et des moyens mis en œuvre pour lutter contre la course et la piraterie, mais également par les différences notables entre ses versions, qui nous renseignent sur le fonctionnement des chancelleries hafside et pisane. Ce traité singulier contraste avec les pratiques diplomatiques en vigueur et surtout avec les principes du droit musulman, qui interdisent à tout souverain de

\footnotetext{
${ }^{50}$ Mas Latrie, Traités 75.

${ }^{51}$ Mas Latrie, Traités 81.
} 
conclure des traités perpétuels avec les puissances chrétiennes. Les Hafsides se démarquent ainsi du reste du monde musulman par leur pragmatisme, les considérations religieuses étant reléguées au second plan au profit d’intérêts économiques.

Si ce traité apparaît à première vue équilibré dans sa globalité, il n’en demeure pas moins que certaines clauses sont contraignantes pour les Pisans ; la responsabilité des consuls est désormais engagée quant aux faits et gestes de leurs concitoyens. Malgré les efforts déployés par la Commune pour se relever et reprendre pied dans le Maghreb, en particulier en Ifrīqiya, en négociant ce dernier traité de paix, le contexte de la fin du $\mathrm{XIV}^{\mathrm{e}}$ siècle montre que la cité toscane est sensiblement affaiblie par ses guerres contre Gênes et par sa lutte contre les Florentins. Ceux-ci s’affirment et finissent par s’emparer de la ville en 1406 ; ils héritent des privilèges pisans qu'ils vont rapidement utiliser pour développer leurs réseaux et lancer leurs galées à la conquête des marchés méditerranéens à partir des années 1420.

\section{Bibliographie}

\section{1- Sources}

- Archivio di Stato di Pisa, Diplomatico, Atti Publici.

- Amari, M., I diplomi arabi del Regio archivio fiorentino, 2 vols., Florence 1863.

- Anonyme, Cronica volgare d'anonimo fiorentino dall'anno 1385 al 1409 già attribuita a Piero di Giovanni Minerbetti, éd. E. Bellondi, Città di Castello 1915 (Rerum Italicarum Scriptores, 27/2).

- Bresc, H. et Rāgib, Y., Le sultan mérinide Abū L-Hasan 'Alī et Jacques III de Majorque. Du traité de paix au pacte secret, Institut français d'Archéologie orientale, Le Caire 2011 (Cahiers des Annales islamologiques, 32).

- Gentile, M. L. (éd.), Gli annales pisani di Bernardo Marangone, Bologne 1930 (Rerum Italicarum Scriptores $\mathrm{VI} / 2$ ).

- Girardi, F., Venezia e il regno di Tunisi. Gli accordi diplomatici conclusi fra il 1231 e il 1456, Rome 2006.

- Ibn ‘Abd al-Barr, Al-Käfì fì fiqh ’ahl al-Madīna, Beyrouth 2012.

- Ibn Khaldūn, Kitāb al-'ibar wa diwān al-mubtada' wa-l-khabar fì tārīkh al-'Arab wa-lBarbar wa man 'āsarahum min thawī al-ša'n al-akbar, éd. Kh. Chahada et S. Zakkar, 7 vols, Beyrouth 1996. 
- Ibn al-Qunfud, Al-Fārisiya fì mabādi' al-dawla al-Fārisiya, éd. M. Ch. Nayfar et A. M. Turki, Tunis 1968.

- Ibn al-Šammā‘, Al-‘adilla al-bayyina al-Nūrāniya fì mafākhir al-dawla al-hafsiya, éd. T. M. Mamouri, Tunis 1984.

- Mas Latrie, L. de., Traités de paix et de commerce de l'Afrique septentrionale avec les nations chrétiennes au Moyen Âge, 2 vols., Paris 1866.

- Al-Qāḍī al-Nu'mān, Da 'ā'im al-'islām, éd. A. Ibn ‘Alī 'Asg̉ar Fayḍ̄i, I, Le Caire 1963.

—, 'Iftitāh al-da'wa, éd. F. Dachraoui, Tunis 1975.

—, Kitāb al-'iqtiṣār, éd. A. Tāmir, Beyrouth 1996.

- Al-Rassā', Šarh hudūd Ibn 'Arafa I, Tunis 2008.

- Tronci, P., Memorie istoriche della citta di Pisa, Livourne 1682.

- Al-Turğumān 'Abd Allâh, Tuhfat al-'arīb fì al-radd 'alā 'ahl al-salīb, éd. O. T. al-Dā'ūq, Beyrouth 1988.

- Al-‘Umarī, Al-ta 'rīf bi-l-muștalah al-šarīf, éd. M. H. Šams al-Dīn, Beyrouth 1988.

\section{2- Études}

- Banti, O., I trattati tra Pisa e Tunisi dal XII al XIV secolo. Lineamenti di storia dei rapporti tra Pisa e il Maghreb, L'Italia ed i paesi mediterranei. Vie di communicazione e scambi commerciali e culturali al tempo delle Reppubliche Marinare, Pise 1988, p. 43-74.

- Bauden, F., Les relations diplomatiques entre les sultans mamelouks circassiens et les autres pouvoirs du Dār al-islām : l'apport du ms. ar. 4440 (BnF, Paris), Annales islamologiques, 41, 2007, p. 1-14.

—, « Due trattati di pace conclusi nel dodicesimo secolo tra i Banū Ġāniya, signori delle isole Baleari, e il comune di Genova », Documentos y manuscritos arabes del Occidente musulman medieval, éd. N. Martinez de Castilla, Madrid 2011, p. 33-86.

- Bensaci, M., Pise et le Maghrib au Moyen Âge, thèse de doctorat inédite, Université Paris X 1979.

- Bresc, H., Un monde méditerranéen, économie et société en Sicile 1350-1450, 2 vols, Palerme-Rome 1986.

- Brunschvig, R., La Berbérie orientale sous les Hafsides des origines à la fin du XV siècle, 2 vols, Paris 1982.

- Doumerc, B., Venise et l'émirat hafside de Tunis (1231-1535), Paris 1999.

- Dufourcq, Ch.-E., L'Espagne catalane et le Maghrib aux XIII et XIV ${ }^{e}$ siècles, Paris 1966. 
- Hamidullah, M., Documents sur la diplomatie musulmane à l'époque du Prophète et des khalifes orthodoxes, Paris 1935.

- Kadduri, M., War and Peace in law of Islam, Baltimore 1955.

- Houssaye Michiensi, I., Datini, Majorque et le Maghreb (14 $-15^{e}$ siècles). Réseaux espaces méditerranéens et stratégies marchandes, Leiden-Boston 2013.

- Ouerfelli, M., Personnel diplomatique et modalités de négociations entre la Commune de Pise et les États du Maghreb (1133-1397), Les relations diplomatiques au Moyen Âge. Formes et enjeux. XLI ${ }^{\mathrm{e}}$ Congrès de la SHMESP, Lyon, 3-6 juin 2010, Paris 2011, p. 119132.

—, «Les ambassadeurs pisans dans le monde musulman (l’Égypte et le Maghreb XII ${ }^{\mathrm{e}}-\mathrm{XIV}^{\mathrm{e}}$ siècle) ", La figure de l'ambassadeur entre mondes éloignés. Ambassadeurs, envoyés officiels et représentants diplomatiques entre Orient islamique, Occident latin et Orient chrétien (XI ${ }^{e}-X V I^{e}$ siècle), dir. N. Drocourt, Rennes 2015, p. 97-110.

-, Diplomatic Exchanges between the City of Pisa and the States of the Maghrib (from the $12^{\text {th }}$ to the $14^{\text {th }}$ century), Mediterranean World, XXII, 2015, p. 97-112.

- Petralia, G., Banchieri e famiglie mercantili nel Mediterraneo aragonese, l'emigrazione dei Pisani in Sicilia nel Quattrocento, Pise 1989.

- Roncioni, R., Delle istorie pisani, libri XVI, Firenze 1844.

- Valérian, D., Bougie, port méditerranéen, 1067-1510, Rome 2006.

—, Le rachat des captifs dans les traités de paix de la fin du Moyen Âge, Hypothèses, 1, 2006, p. 343-358.

—, Les relations entre l'Italie et le Maghreb dans la seconde moitié du $\mathrm{XV}^{\mathrm{e}}$ siècle. Les conditions d'un nouvel essor, Maghreb-Italie. Des passeurs médiévaux à l'oerientalisme moderne (XIII ${ }^{e}$-milieu du XX $X^{e}$ siècle), éd. Benoît Grévin, Rome, École française de Rome, 2010, p. 85-102.

\section{Annexes}

1- Archivio di Stato di Pisa, Diplomatico, Atti Pubblici : 23 rabī‘ al-’awwal 800/14 décembre 1397 (n. s.) : traité de paix et de commerce conclu entre le sultan hafside Abū Fāris 'Abd al-'Az̄̄z et le capitaine de Pise Giacomo d'Appiano, par l'intermédiaire de l'ambassadeur Andrea di Michele del Campo.

2- ASP. Diplomatico. Atti Publici, cartaceo, 14 décembre 1397 (n. s.), version italienne, exemplaire $C$, détail. 